\title{
Link between Mass-loss and Variability Type for AGB Stars?
}

\author{
Željko Ivezić, Gillian R. Knapp \\ Princeton University, Department of Astrophysical Sciences, Princeton, \\ NJ 08544-1001, USA
}

\begin{abstract}
.
We find that AGB stars separate in the $25-12$ vs. $12-\mathrm{K}$ color-color diagram according to their chemistry $(\mathrm{O}, \mathrm{S}$ vs. $\mathrm{C})$ and variability type (Miras vs. SRb/Lb). While discrimination according to the chemical composition is not surprising, the separation of Miras from SRb/Lb variables is unexpected.

We show that "standard" steady-state radiatively driven models provide excellent fits to the color distribution of Miras of all chemical types. However, these models are incapable of explaining the dust emission from O-rich SRb/Lb stars. The models can be altered to fit the data by postulating different optical properties for silicate grains, or by assuming that the dust temperature at the inner envelope radius is significantly lower $(300-400 \mathrm{~K})$ than typical condensation temperatures $(800-1000 \mathrm{~K})$, a possibility which is also supported by the detailed characteristics of LRS data. While such lower temperatures are required only for $\mathrm{O}$ - and $\mathrm{S}$-rich SRb/Lb stars, they are also consistent with the colors of $\mathrm{C}$-rich $\mathrm{SRb} /$ Lb stars.

The absence of hot dust for $\mathrm{SRb} / \mathrm{Lb}$ stars can be interpreted as a recent (order of $100 \mathrm{yr}$ ) decrease in the mass-loss rate. The distribution of O-rich $\mathrm{SRb} / \mathrm{Lb}$ stars in the 25-12 vs. K-12 color-color diagram shows that the mass-loss rate probably resumes again, on similar time scales. It cannot be ruled out that the mass-loss rate is changing periodically on such time scales, implying that the stars might oscillate between the Mira and SRb/Lb phases during their AGB evolution as proposed by Kerschbaum et al. (1996). Such a possibility appears to be supported by recent HST images of the Egg Nebula obtained by Sahai et al. (1997), the discovery of multiple CO winds reported by Knapp et al. (1998), and long-term visual light-curve changes detected for some stars by Mattei (1998).
\end{abstract}

\section{Introduction}

AGB stars are long-period variables (LPV) and show a variety of light curves. Based on visual light curves, the General Catalog of Variable Stars (GCVS) defines regular variables, or Miras, semiregular (SR) variables, and irregular variables $(\mathrm{L})$. The distinctive features are the regularity of light curves, their 
amplitude and their period. Some types are further subdivided based on similar criteria (e.g. SRa, SRb, SRc,...).

AGB stars of different variability types cannot be distinguished by considering IRAS PSC data alone. Kerschbaum, Hron and collaborators (Kerschbaum \& Hron 1996, and references therein; hereafter KH) have studied IR emission of various types of LPVs by combining IRAS broad-band fluxes with near-IR observations and spectra from the IRAS LRS database. They find that SRa variables appear to be a mixture of two distinct types: Miras and SRb variables. $\mathrm{SRb}$ variables have somewhat higher stellar temperatures and smaller optical depths than Miras, and can be further divided into "blue" and "red" subtypes, the former showing much less evidence for dust emission than the latter.

Particularly intriguing are differences between the LRS spectra of SRb variables and Miras with " 10 " $\mu$ m silicate emission feature (LRS class $2 \mathrm{n}$ ). The peak position of the "10" $\mu \mathrm{m}$ feature for SRb sources is shifted longwards, relative to the peak position for Miras, by $0.2-0.3 \mu \mathrm{m}$. In addition, the ratio of the strengths of silicate emission features at $18 \mu \mathrm{m}$ and $10 \mu \mathrm{m}, F_{18} / F_{10}$, is larger for SRb variables than for Miras. These results are confirmed at high statistical significance by Marengo (1998), who determines the " $10 " \mu \mathrm{m}$ feature peak position by fitting a polynomial to the $9-11 \mu \mathrm{m}$ spectral region. He finds a difference in median peak positions of $0.2 \mu \mathrm{m}$, with probability larger than 0.9999 that the two distributions are different.

Motivated by these results, we further analyze correlations between IR emission and variability type for AGB stars, and their implications for the models of steady-state radiatively driven outflows. We base our analysis on IRAS data and a set of JHKLM photometric observations for about $600 \mathrm{LPVs}$, kindly made available to us by Franz Kerschbaum.

\section{Distribution of AGB stars in K-12-25 Color-color Diagram}

Following the results of $\mathrm{KH}$, we consider only SRb, Lb, and Mira variables, and further divide them according to grain chemistry. Fig. 1 displays the distribution of sources in the K-12-25 diagram ${ }^{1}$.

Stars in our sample clearly separate according to grain chemistry ( $\mathrm{O}$ vs. $\mathrm{C}$ stars, $\mathrm{S}$ stars cannot be distinguished from $\mathrm{O}$ stars). Such a clear separation is not very surprising since the optical properties of silicate and carbon grains, which by and large determine the spectral shape of dust emission, are significantly different at these wavelengths. Stars also separate according to their GCVS variability type into two well defined regions, Miras and non-Miras (i.e. $\mathrm{SRb}$ and $\mathrm{Lb}$, which exhibit similar behavior). This is in sharp contrast with the distribution of the same sample in the IRAS 12-25-60 diagram, which shows no such division.

A scheme which classifies stars into 4 groups according to their variability type (Miras, $\mathrm{m}$, vs. non-Miras, $\mathrm{n}$ ) and grain chemistry ( $\mathrm{O}$ stars with silicate grains and $\mathrm{C}$ stars with carbonaceous grains) is shown in Fig. 1 by solid lines.

\footnotetext{
${ }^{1}$ We use colors defined as $\lambda_{2}-\lambda_{1}=\log \left(F_{\nu}\left(\lambda_{2}\right) / F_{\nu}\left(\lambda_{1}\right)\right)$, where $F_{\nu}\left(\lambda_{1}\right)$ and $F_{\nu}\left(\lambda_{2}\right)$ are fluxes at wavelengths $\lambda_{1}$ and $\lambda_{2}$.
} 


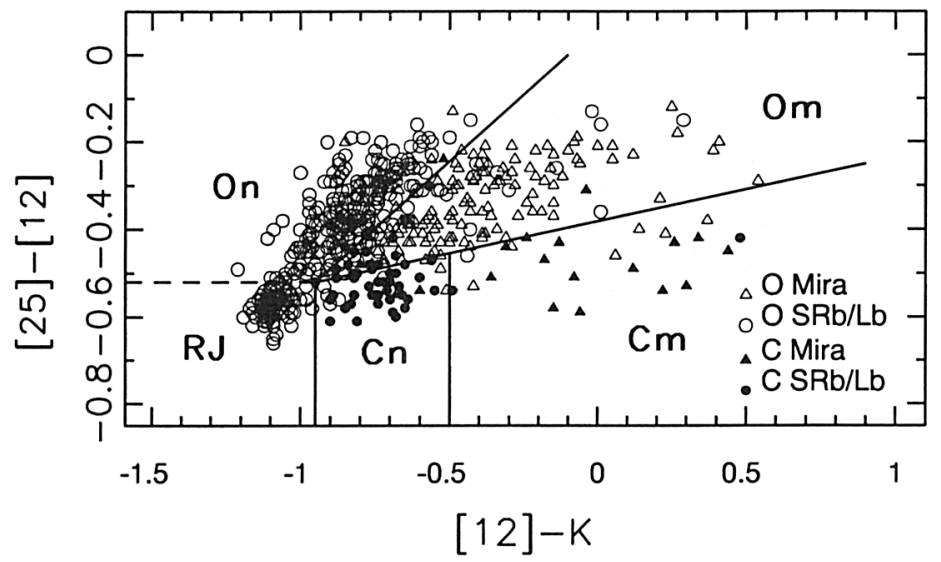

Figure 1. K-12-25 color-color diagram for mass losing AGB stars. Various symbols correspond to source variability type and grain chemistry, as marked. Solid lines display classification into 4 regions according to grain chemistry $(\mathrm{O}$ and $\mathrm{S}$ stars with silicate grains vs. $\mathrm{C}$ stars with carbonaceous grains), and variability type (Miras, $m$, vs. nonMiras, n). Dashed line separates On sources near the Rayleigh-Jeans point (RJ) which have no, or very little dust.

The boundaries, required to be straight lines, are determined by maximizing the efficiency and reliability of the resulting classification. The dashed line separates On sources around the Rayleigh-Jeans point and is determined by assuming a comparable scatter in both colors.

\section{Interpretations of the Source Distribution in K-12-25 Color-color Diagram}

\subsection{Predictions of the "Standard" Models}

We first attempt to model the source distribution by employing spectra expected for a steady-state radiatively driven outflow with either silicate or amorphous carbon grains (Ivezić \& Elitzur 1995, and references therein). We define "standard" models by $2500 \mathrm{~K}$ black body stellar spectral shape, and dust temperature at the inner envelope edge of $1200 \mathrm{~K}$ for carbon grains and $700 \mathrm{~K}$ for silicate grains. These two temperatures mark the "standard" model tracks in Fig. 2. For each type of grain, the model track starts at the Rayleigh-Jeans point, with the positions along the track parametrized by dust optical depth.

The track for carbon grains passes through the distribution of stars with carbon dust, i.e. through the regions $\mathrm{Cn}$ and $\mathrm{Cm}$. The scatter of points is marginaly consistent with the photometric errors, and non-Mira stars appear to be smaller optical depth counterparts of Miras. The model track for silicate dust passes through the distribution of Miras in Om region, although not through the region with the highest density of sources. The offset of about 0.5 mag. $(0.2$ on plotted log scale) is somewhat large, but still not sufficient to clearly rule 


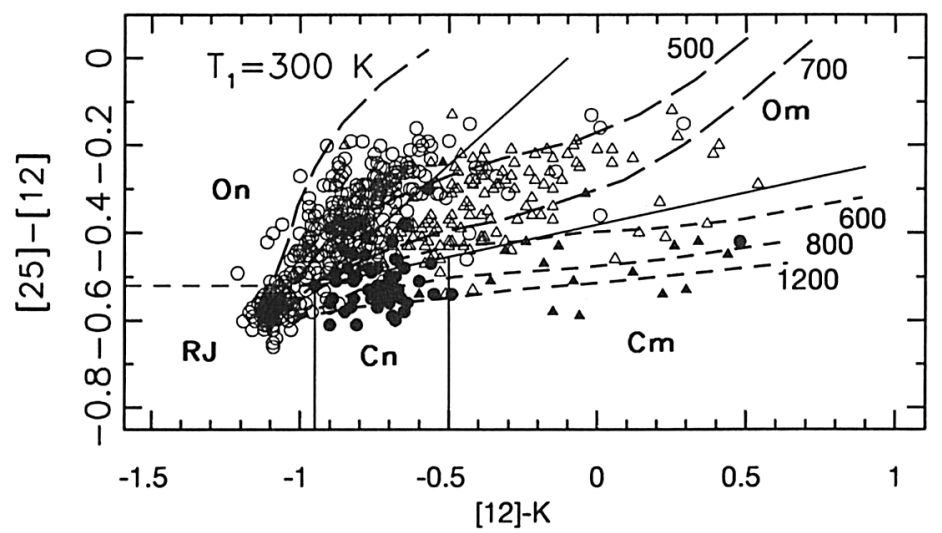

Figure 2. K-12-25 color-color diagram for mass losing AGB stars. Symbols correspond to source variability type and grain chemistry, as marked in Fig. 1. Long-dashed lines are model tracks for silicate grains with various dust temperatures at the inner envelope radius, $\mathrm{T}_{1}$, as marked in the figure. Models for amorphous carbon grains are displayed by short-dashed lines. "Standard" models have $\mathrm{T}_{1}=700 \mathrm{~K}$ (silicate grains) and $\mathrm{T}_{1}=1200 \mathrm{~K}$ (carbon grains).

out the model. However, the model prediction is in dire disagreement with the distribution of non-Miras with silicate dust (region On). For example, for 25-12 color of -0.3 , the discrepancy in $12-\mathrm{K}$ color is about 0.75 on log scale, or almost 2 magnitudes.

\subsection{Variations of the "Standard" Models}

There are several ways to augment the "standard" models without significantly changing the paradigm of steady-state radiatively driven outflow.

Stellar spectral shape can effect the model spectrum when dust optical depth is not large. For example, increasing the stellar temperature moves a model track to the left in the K-12-25 color-color diagram, as required by the data. However, we are unable to produce satisfactory model tracks by varying the stellar temperature within a plausible range $(<4000 \mathrm{~K})$. Another possibility is that a black-body spectral shape is not an adequate description of the true stellar spectrum. This can be ruled out by considering the K-L-M color-color diagram: differences in median near-IR colors between Miras and non-Miras are about 3-4 times smaller than required to account for the observed differences in $12-\mathrm{K}$ color. Such small differences in near-IR data also rule out an absorption feature in the $\mathrm{K}$ band, e.g. $\mathrm{H}_{2} \mathrm{O}$ feature as seen in the data by Matsuura et al. (1998), as an explanation for redder 12-K colors observed for Miras. Furthermore, similar separation of stars into 4 regions can also be seen in the L-12-25 color-color diagram.

Models in agreement with data for non-Miras can be produced by altering the spectral shape of absorption efficiency for silicate grains. There are two required changes: the ratio of the strengths of silicate features at $18 \mu \mathrm{m}$ and $10 \mu \mathrm{m}$ has to be increased by a factor of about $2-3$, and the peak position of 


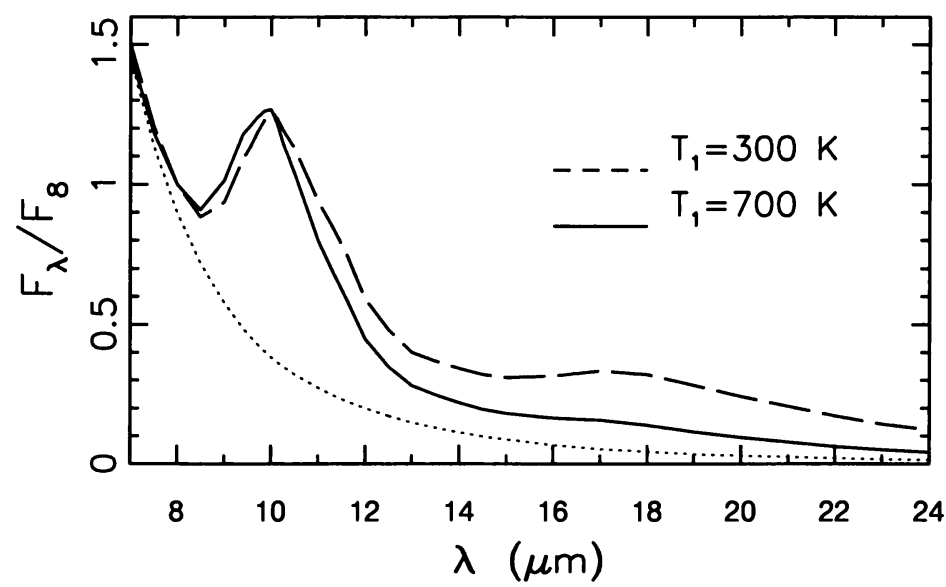

Figure 3. Model spectra in the LRS region obtained for silicate grains and 2 values of the dust temperature at the inner envelope radius, $\mathrm{T}_{1}$ (solid and dashed lines). The dotted line shows the stellar contribution to the spectra. Note the horizontal shift between the features, although both are calculated with the same grain optical properties.

the " 10 " micron feature has to be shifted longwards by about $0.2-0.3 \mu \mathrm{m}$. Such absorption efficiency results in a model track which leaves the Rayleigh-Jeans point at a larger angle than before, and passes exactly through the observed source distribution.

Another way to make "standard" models agree with the data is to change $\mathrm{T}_{1}$, the assumed dust temperature at the inner envelope edge. The model tracks for (unaltered) silicate grains with $\mathrm{T}_{1}=300,500$ and $700 \mathrm{~K}$ are shown in Fig. 2 by solid lines. They demonstrate that the distribution of stars with silicate dust in the K-12-25 color-color diagram can be reproduced by varying $T_{1}$ in the range 300-700 K, without any change in the adopted absorption efficiency for silicate grains. It is remarkable that these models are also capable of explaining the differences between Miras and non-Miras found in LRS data. Fig. 3 displays two model spectra obtained with visual optical depth of 0.6 , and $T_{1}=300 \mathrm{~K}$ (dashed line) and $700 \mathrm{~K}$ (solid line). The dotted line shows the stellar spectrum. Because the hot dust has been removed in the model with $\mathrm{T}_{1}=300 \mathrm{~K}$, both the blue and red edges of the silicate $10 \mu \mathrm{m}$ emission feature are shifted longwards by about $0.2-0.3 \mu \mathrm{m}$, causing the whole feature to appear shifted although the peak position is the same in both models. In addition, this model has a larger $F_{18} / F_{10}$ flux ratio, as observed. This success and the application of Occam's razor hints that the absence of hot dust is the most likely explanation for differences in IR spectra between Mira and non-Mira stars with silicate dust.

The distribution of Miras and non-Miras with carbon dust is fairly well described by the "standard" model with $\mathrm{T}_{1}=1200 \mathrm{~K}$, and thus the assumption of significantly lower $T_{1}$ is not required by the data. However, such models are not ruled out by the data as shown by model tracks for $\mathrm{T}_{1}=600,800$ and 1200 $\mathrm{K}$ displayed by dashed lines in Fig. 2. 


\section{Discussion}

Absence of hot dust, as implied by low $\mathrm{T}_{1}$, might be a natural consequence of a significant recent decrease in mass-loss rate. From the distribution of sources in the On region, we infer a time scale of about 100 years. What happens after that? The distribution of On sources ends quite sharply at its blue edge. If envelopes continued to freely expand, distribution would extend smoothly to bluer $12-\mathrm{K}$, and redder 25-12 colors. The absence of these sources argues that mass-loss resumes after about 100 years. This idea, that stars oscillate between the SRb and Mira phases, was proposed by Kerschbaum et al. (1996) based on similar number densities and scale heights.

This possibility seems to be supported by a number of new observations. Recent HST images of the Egg Nebula obtained by Sahai et al. (1997) show concentric shells whose spacing corresponds to a dynamical time scale of about 100 years. The discovery of multiple CO winds reported by Knapp et al. (1998) is in agreement with the hypothesis of variable mass-loss. Furthermore, they find that the velocity and mass-loss rate for each individual component follows the relation $v \propto \dot{M}^{1 / 3}$ found for stars with single CO winds (Young 1995), and theoretically expected for radiatively driven outflows (Ivezić, Knapp \& Elitzur 1998). Finally, there is mounting direct evidence that long-term visual light curve changes are consistent with the transition between Mira and non-Mira variability types (Mattei 1998).

If proven correct, the mass-loss variations on time scales of about 100 years would significantly change our understanding of the stellar evolution on the AGB. What type of observations could rule out this hypothesis? The most obvious and direct test is mid-IR imaging. When $T_{1}$ decreases by a factor of two, the inner envelope radius increases by about a factor of four. Such a difference should be easily discernible for a few dozen candidate stars with largest angular sizes, which are already within the reach of the Keck telescopes (Monnier 1999).

Acknowledgments. We are grateful to Franz Kerschbaum for his JHKLM observations without which this work would not have been possible. Massimo Marengo and Mikako Matsuura have endebted us by communicating their results prior to publication. This work was supported by NSF grant AST96-18503.

\section{References}

Ivezić Ž., Elitzur M., 1995, ApJ 445, 415

Ivezić Ž., Knapp G.R., Elitzur M., 1998, astro-ph/9805003

Kerschbaum F., Hron J., 1996, A\&A 308, 489

Knapp G.R., Young K., Lee E., Jorissen A., 1998, ApJS 117, 209

Marengo M., 1998, in preparation

Matsuura M., et al., 1998, in preparation

Mattei J.A., 1998, private communication

Monnier J., 1999, this volume

Sahai R., Trauger J.T., Watson A.M., et al., 1998, ApJ 493, 301

Young K., 1995, ApJ 445, 872 